\title{
Difficulties in diagnosing HIV-associated nephropathy in kidney transplanted patients. The role of ultrasound and CEUS.
}

\author{
Florin Ioan Elec ${ }^{1,2}$, Tudor Moisoiu', Mihai Adrian Socaciu ${ }^{3}$, Alina Daciana Elec ${ }^{4}$, Adriana \\ Milena Muntean ${ }^{4}$, Gheorghiță Iacob ${ }^{5}$, Radu Ion Badea ${ }^{3}$
}

${ }^{1}$ Urology Department, Clinical Institute of Urology and Kidney Transplant, ${ }^{2 “ I u l i u ~ H a t i e g a n u ” ~ U n i v e r s i t y ~ o f ~ M e d i-~}$ cine and Pharmacy, ${ }^{3}$ Ultrasound Department, "Octavian Fodor" Institute of Gastroenterology and Hepatology, "Iuliu Hatieganu" University of Medicine and Pharmacy, ${ }^{4}$ Nephrology Department, Clinical Institute of Urology and Kidney Transplant, ${ }^{5}$ Morphology Department, Clinical Institute of Urology and Kidney Transplant, Cluj Napoca, Romania

\footnotetext{
Abstract

A 62-year-old woman who underwent kidney transplantation in 2014 was diagnosed with HIV infection in 2018. Grey scale and Doppler ultrasound evaluation revealed a normal aspect of the allograft. Contrast-enhanced ultrasound detected a quick cortical contrast uptake followed by a rapid cortical wash-out. This behavior was interpreted as a sign of inflammation. Ten months after ultrasound evaluation the graft presented severe disfunction and the patient was reintroduced into the hemodialysis program.
}

Keywords: Contrast-enhanced ultrasound; HIV infection; allograft; kidney

\section{Introduction}

The increasing number of solid organ transplantation (SOT) and the extension of donor and recipient approval criteria has lead to new challenges in the follow-up. One emerging issue is the HIV infection among solid organ transplant patients. The excellent control of HIV infection after the introduction of high activity antiretroviral therapy has lead to the approval of HIV infected patients to receive solid organ transplant. In the field of renal transplantation, good results were reported in the USA and Europe, HIV infected patients having a similar outcome $[1,2]$.

Received 22.11.2019 Accepted 11.12.2019

Med Ultrason

2019:0 Online first, 1-4

Corresponding author: Tudor Moisoiu, MD

Clinical Institute of Urology and Kidney

Transplant, 4-6 Clinicilor street

400006, Cluj-Napoca, Romania

E-mail: tmoisoiu@gmail.com

Phone: +4 0752625989
Despite this, there is not much in the literature that describes patient management of HIV infection after kidney transplantation, considering this is a rare situation. We present the case of a 62-year-old woman who underwent kidney transplantation in 2014 beeing diagnosed with HIV in 2018. In 2019 the graft was lost and the patient was returned to hemodialysis.

\section{Case report}

A 62-year-old woman, with end-stage renal disease and hemodialysis for one year, received in 2014 a kidney transplant from a deceased donor. Following internal protocols, immunosuppressant therapy was started, including Advagraf, Myfortic and Prednisone. After transplantation, she had a slow graft recovery, with significant improvement after six months follow-up, with a baseline serum creatinine $(\mathrm{sCr})$ of $1.28 \mathrm{mg} / \mathrm{dL}$, urea 59 $\mathrm{mg} / \mathrm{dL}$ and hemoglobin of $11 \mathrm{~g} / \mathrm{dL}$. From May 2017 till September 2017 the patient presented progressive graft dysfunction with a serum creatinine of $2.49 \mathrm{mg} / \mathrm{dL}$, pro- 
teinuria at nephritic level $(2 \mathrm{~g} / 24 \mathrm{H})$, persistent leucopenia and positive Epstein-Barr serology tests. Allograft biopsy was indicated, but it was delayed because of an Entoroccocus feacalis urinary tract infection. Aciclovir and antibiotics were administrated. In February 2018 the serum creatinine was $2.89 \mathrm{mg} / \mathrm{dl}$, proteinuria $4 \mathrm{~g} / 24 \mathrm{H}$, leukocyte 3800/uL. Allograft biopsy was performed proving tubulointerstitial nephritis (TIN) and Banff 1A acute rejection. Prednisone dose was increased with the stabilization of the graft function. In June 2018 allograft function was stable, $\mathrm{sCr} 2.39 \mathrm{mg} / \mathrm{dL}$ and the patient was included in an observational study where extensive allograft ultrasonography (US) was performed.

Grey scale US showed normal parenchymal thickness but an increased cortico-medullar contrast due to the hyperechoic cortex and hypoechoic medulla. Color Doppler US had a normal appearance (fig 1). Contrastenhanced US (CEUS) examination showed early and late pyelographic phases, quick cortical contrast uptake (at $15 \mathrm{~s}$ ), normal medullar enhancement (at $25 \mathrm{~s}$ ) and quick cortical wash-out (at $50 \mathrm{~s}$ ) (fig 2). Quantification using VueBox (Bracco) with ROIs at the level of the cortex, medulla and interlobar arteries with the resulting TIC (time-intensity curves), confirmed the early wash-out of the cortex, probably in the context of inflammation (fig 3). Parametric maps based on Peak enhancement $(\mathrm{PE})$, area under the wash-in and wash-out curve (AUC) and time to peak (TTP) also showed a relatively low AUC in the cortex (similar to the medulla), despite the increased PE and normal TTP, due to a quick washout (fig 4). The findings were in correlation with the pathology result of TIN.

In September 2018 the patient was admitted with bronchopneumonia and persistent neutropenia. The extensive test proved that the patient had HIV infection with 356.000 copies, CD4 $20 / \mathrm{mm}^{3}$. At this point, $\mathrm{sCr}$ reached $5 \mathrm{mg} / \mathrm{dL}$. Antiretroviral treatment was initiated with Dolutegravir $50 \mathrm{mg} /$ day and Lamivudine $100 \mathrm{mg} /$ day. Because the patient was not able to point to a cause of the infection, serum testing for HIV from the serum bank was preceded. The first positive test was obtained in September 2017. The other allograft recipient was tested, but it was negative, showing that the infection was probably sexually acquired. At six months CD4 was $319 / \mathrm{mm}^{3}$ with undetectable viremia, but graft function continued to decline, the right serum concentration of immunosuppressant and antiretroviral drugs being unable to reach due to complex interactions; in March 2019 the patient reentered chronic hemodialysis.

\section{Discussion}

This case highlights the diagnostic challenges of post-transplantation HIV infection with a probable sexual acquisition. This issue is not well described in the literature, only one case series being available from our research [3]. Even though the benefits of transplantation in HIV infected patients has been well known for years,
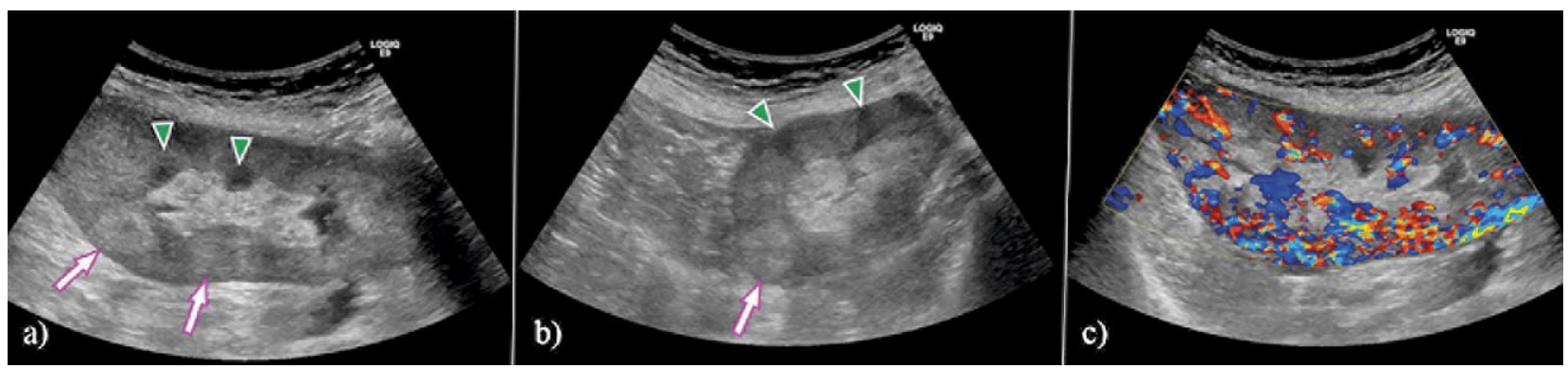

Fig 1. B-mode scan in longitudinal (a) and transverse (b) plane with the hyperechoic cortex (long arrow) and hypoechoic medulla (arrowhead). Normal appearance in color Doppler mode (c).
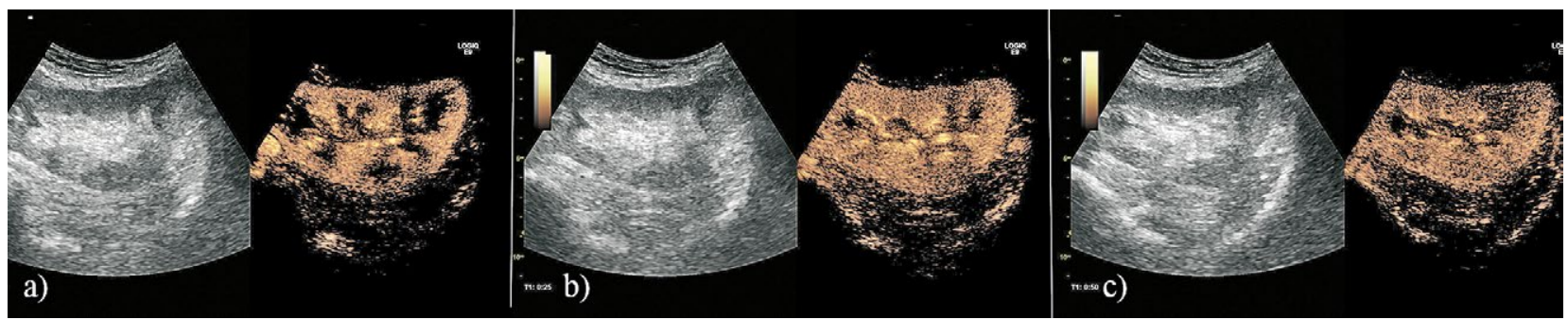

Fig 2. CEUS enhancement in the cortico-medullar (a), early pyelographic (b), and late pyelographic (g) phases show a quick cortical contrast uptake and quick cortical wash-out. 


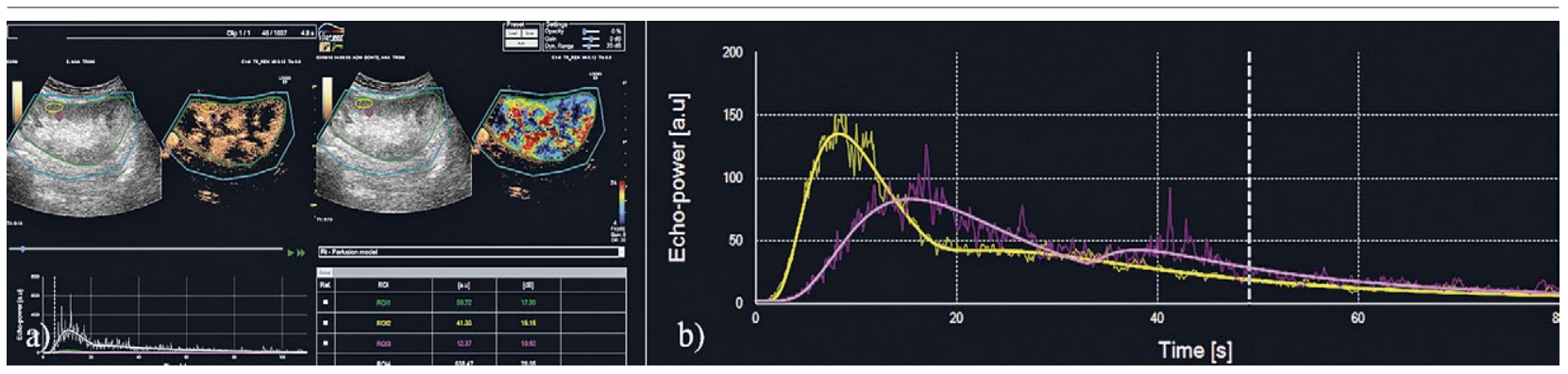

Fig 3. Quantification using VueBox (Bracco) with region of interest (ROI) at the level of the cortex, medulla and interlobar arteries (a) with the resulting time intensity curves (TIC) (b); yellow = cortex; magenta = medulla. The curve confirms the early wash-out of the cortex, probably in the context of inflammation.

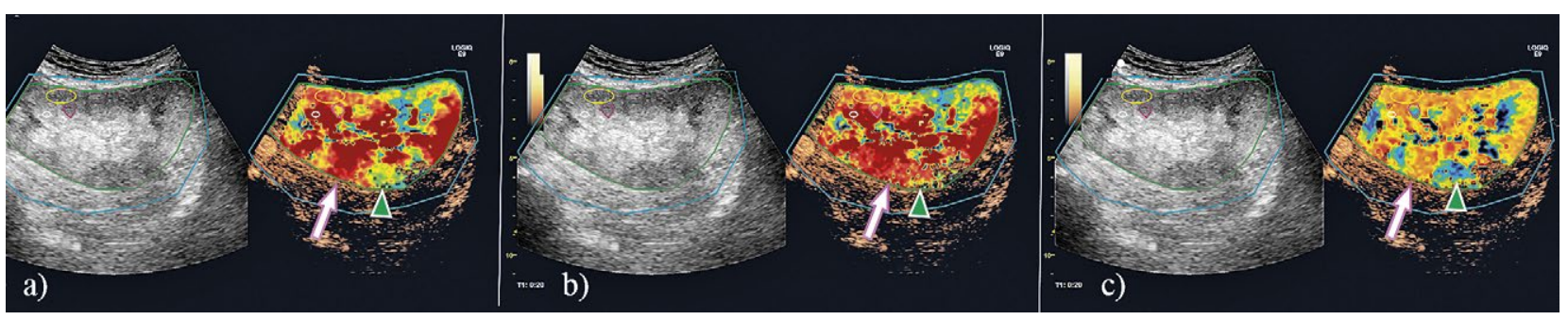

Fig 4. Parametric maps based on peak enhancement (PE) (a), area under the wash-in and wash-out curve (AUC) (b), and time to peak (TTP) (c) also show a relatively low AUC in the cortex.

there is little data on the workup and management of HIV infection after lengthy periods after SOT.

This case is an unusual case of acute renal allograft dysfunction that at first seemed to be an Epstein-Barr infection, but with worsening clinical evolution after Aciclovir treatment. Following the allograft biopsy, the diagnostic of Banff 1A acute rejection and TIN shifted the treatment to increase corticoid treatment, with the stabilization of the graft function for about three months. At this point, the biopsy result was considered an explanation for acute allograft dysfunction, neutropenia being considered a side effect of immunosuppressive treatment. The pathology result was misleading because of the absence of tubuloreticular aggregates in endothelial cells, a pathognomonic feature of the HIV-associated nephropathy (HIVAN). The aspect of TIN and collapsing glomerulopathy can be found in viral nephropathy, calcineurin inhibitors toxicity and vascular disease (thrombotic microangiopathy), making the diagnosis of HIVAN in a transplanted patient very difficult [4].

US is one of the most cost-effective imaging methods used in nephrology and SOT. Despite this, there are not many studies about US aspects of HIVAN. Unfortunately, the US diagnostic of HIVAN is strongly related to prior diagnostic of HIV because of the lack of specific signs. Imaging findings that are suggestive of HIVAN and have been previously reported include normal-sized or enlarged kidneys, increased cortical echogenicity, renal pelvicalyceal thickening, and loss of the renal sinus fat appearance [5]. At the same time, acute allograft rejection has a similar US appearance with increased cortical echogenicity and hypoechogenic medulla [6]. Only the use of CD4+ or CD8+ labeled microbubbles was proved in rats to be able to detect cell-mediated acute rejection [6]. In our case, CEUS examination revealed a fast washout of contrast suggesting allograft inflammation, in consensus with the diagnostic of TIN. To our knowledge, this is the first case of a CEUS examination for HIVAN.

Only the onset of bronchopneumonia associated with neutropenia suggested HIV infection. At the time of diagnosis, the CD4 count was $20 / \mathrm{mm}^{3}$ and a viral load of 356.000 copies with the meaning of advanced disease and high risk of HIVAN [7]. Retrospectively, we consider that persistent neutropenia, prolonged allograft dysfunction with unresponsive to treatment, AR and TIN diagnosed at biopsy and CEUS findings of allograft inflammation were suggestive for HIV infection and HIVAN.

In conclusion, in a case of renal graft dysfunction with persistent neutropenia where there is the suspicion of AR and TIN after allograft biopsy, with CEUS suggestive for inflammation, HIV nephropathy as a possible cause of renal graft impairment and graft loss should be take in consideration.

Acknowledgments: This work was supported by a CNCSIS - UEFISCDI grant, project number PN-III-P4ID-PCE-2016-0701, with PNCDI II, contract number: $184 / 2017$ 


\section{References}

1. Mazuecos A, Fernandez A, Andres A, et al. HIV infection and renal transplantation. Nephrol Dial Transplant 2011;26:1401-1407.

2. Stock PG, Barin B, Murphy B, et al. Outcomes of kidney transplantation in HIV-infected recipients. N Engl J Med 2010;363:2004-2014.

3. Cristelli MP, Mazolin MA, Manzardo C, et al. Sexual acquisition of HIV infection after solid organ transplantation: Late presentation and potentially fatal complications. Transpl Infect Dis 2018;20: e12894.
4. Medapalli RK, He JC, Klotman PE. HIV-associated nephropathy: Pathogenesis. Curr Opin Nephrol Hypertens 2011;20:306-311.

5. Symeonidou C, Standish R, Sahdev A, Katz RD, Morlese J, Malhotra A. Imaging and histopathologic features of HIVrelated renal disease. Radiographics 2008;28:1339-1354.

6. Jehn U, Schuette-Nuetgen K, Kentrup D, Hoerr V, Reuter S. Renal Allograft Rejection: Noninvasive Ultrasound- and MRI-Based Diagnostics. Contrast Media Mol Imaging 2019;2019:3568067.

7. Wojciechowski D, Gandhi RT, Rosales IA. Case 11-2019: A 49-Year-Old Man with HIV Infection and Chronic Kidney Disease. N Engl J Med 2019;380:1464-1472. 